\title{
Transport in Double-Crossed Luttinger Liquids
}

MRI-PHY/P981168

\author{
P. Durganandini円 \\ Department of Physics, Pune University, \\ Pune $41100 \%$, India. \\ Sumathi Rao \\ Mehta Research Institute, Chhatnag Road, Jhunsi, \\ Allahabad 211 019, India.
}

\begin{abstract}
We study transport through two Luttinger liquids ( one-dimensional electrons interacting through a Coulomb repulsion in a metal) coupled together at two points. External voltage biases are incorporated through boundary conditions. We include density-density couplings as well as single-particle hops at the contacts. For weak repulsive interactions, transport through the wires remains undisturbed by the inter-wire couplings, which renormalise to zero. For strong repulsive interactions, the inter-wire couplings become strong. For symmetric barriers and no external voltage bias, a single gate voltage is sufficient to tune for resonance transmission in both wires. However, for asymmetric couplings or for finite external biases, the system is insulating.
\end{abstract}

PACS numbers: 71.10.Pm, 72.10.-d, 73.40.Gk

\footnotetext{
${ }^{1}$ e-mail address: pdn@physics.unipune.ernet.in

2 e-mail address: srao@thwgs.cern.ch, sumathi@mri.ernet.in
} 
Interest in the study of one-dimensional systems and Luttinger liquid behaviour [1] has remained high ever since the discovery of high $T_{c}$ superconductors and Anderson's suggestion [2] that they could be explained by two-dimensional Luttinger liquids. But the study of onedimensional systems and Luttinger liquids has received a boost in recent years, since it was found that it was actually possible to fabricate one-dimensional quantum wires operating in the single channel limit [3]. Other experimentally realisable Luttinger liquid systems were the edge states in the fractional quantum Hall bar [4], carbon nanotubes [5] and long chain molecules [6].

Although still beset with contact problems, it has now become clear that transport measurements in such systems could directly probe Luttinger liquid behaviour in the near future. A detailed paper by Kane and Fisher[7] a few years ago addressed the question of transport through wires with one or two weak barriers or, in the opposite limit, through one or two weak links. This has led to a lot of further work in this area, with inclusion of external voltage biases [8, 9], exact solutions at a particular value of the Luttinger liquid coupling parameter [10] and finally the exact solution at arbitrary coupling parameter using the thermodynamic Bethe ansatz11.

In a recent paper, Komnik and Egger 12] studied crossed Luttinger liquids - i.e., two wires (modelled by spinless Luttinger liquids) coupled at a point. External potential biases were introduced through boundary conditions [8] on the densities of the left and right-movers and inter-wire density-density coupling and single particle hoppings were considered. Interestingly, they found that the current through each wire was sensitive to the cross-voltage ( the voltage drop across the other wire). This is contrary to what is expected in an uncorrelated system and was hence a sensitive test of Luttinger liquid behaviour.

In this paper, we extend this work and study doubly crossed Luttinger liquids, or two wires coupled at two points. We also incorporate external biases through boundary conditions and consider density-density and single particle hoppings. We find that for weak repulsive Coulomb interactions, transport through both the wires remain undisturbed by the inter-wire couplings. For strong repulsions, the inter-wire couplings grow and become opaque to transmission. This fixes the charge on the island between the two coupling points. Interestingly however, for symmetric couplings between the wires, and no external voltage bias, a single gate voltage is sufficient to tune for resonant transmission in both wires. For asymmetric couplings or with external voltage biases, the system is insulating, except when the value of the Luttinger liquid coupling is $g=1 / 2$. At this particular value of $g$, even asymmetric couplings or finite external biases, lead to resonant transmission, albeit less than perfect.

We start with two quantum wires with one-dimensional left and right moving fermions. At the ends of the wire, we have external ideal reservoirs (a la Landauer), held at potentials $U_{i}^{A}, i=1,2$ at the left and right edges and $A=1,2$ for the two wires. $\left(U_{1}^{A}-U_{2}^{A}\right.$ is the applied two terminal voltage in each wire.) as shown in Fig.1. 


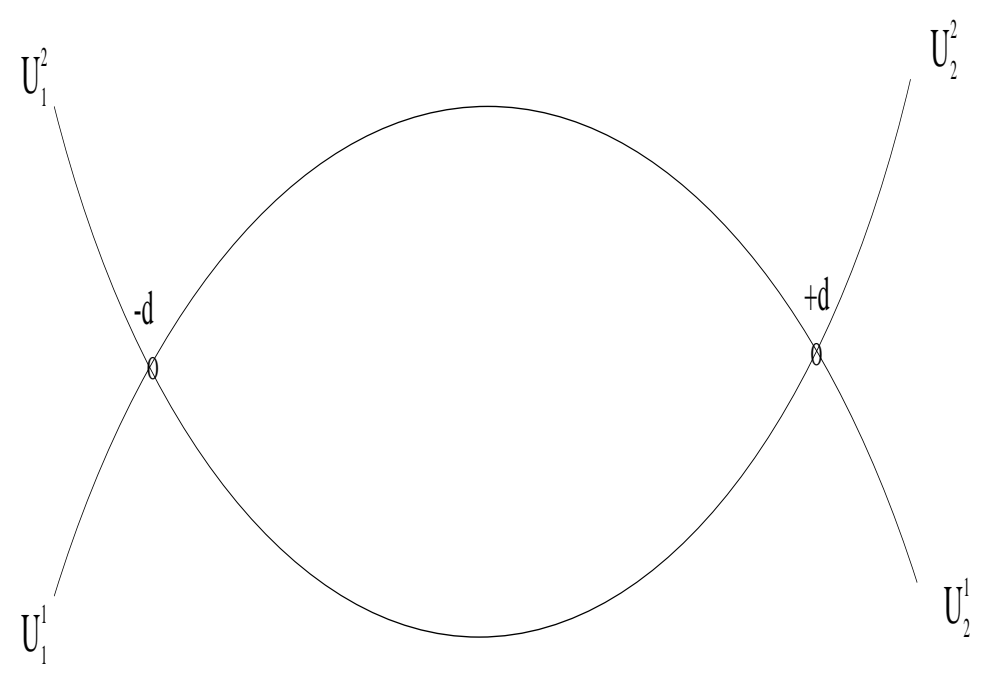

- Fig 1. Two Luttinger liquids coupled together at two points $(x=-d$ and $x=+d)$ and connected to external reservoirs held at constant voltages $U_{1}^{1}, U_{1}^{2}$ on the left and $U_{2}^{1}, U_{2}^{2}$ on the right.

The two wires are coupled together at two points located at $-d$ and $d$. We introduce screening backgates around the quantum wire at a distance $R_{s}$, to screen the Coulomb interaction. $R_{s}<<L$, where $L$ is the length of the wire, but is much larger than the diameter of the quantum wire, which we take to be vanishingly small.

We specialise to the case of spinless fermions and use the standard methods of abelian bosonisation (for a detailed comparison of different bosonisation methods, see Ref. [13]) to write the Hamitonian for each of the uncoupled wires in terms of a scalar field as

$$
H=\frac{\hbar v_{F}}{2 g} \sum_{A=1}^{2} \int d x\left[g\left(\partial_{x} \phi^{A}\right)^{2}+g^{-1}\left(\partial_{x} \theta^{A}\right)^{2}\right]
$$

where the fields $\phi^{A}$ and $\theta^{A}$ are related to the right and left-moving fermion fields as

$$
\psi_{p}^{A}(x)=\frac{1}{(2 \pi a)^{1 / 2}} \exp \left[-i p k_{F} x-i \sqrt{\pi} \phi^{A}(x)-i p \sqrt{\pi} \theta^{A}(x)\right]
$$

where $p=+/-$ stands for $R / L$ movers and $g$ is the Luttinger liquid parameter. For future use, we note that the boson representation for the electron density is given by

$$
\rho^{A}(x)=\frac{1}{\sqrt{\pi}} \partial_{x} \theta^{A}(x)+\frac{1}{\pi a} \cos \left(2 k_{F} x+2 \sqrt{\pi} \theta^{A}(x)\right)
$$

where we have dropped the equilibrium density $\rho=k_{F} / \pi$, (which is expected to be neutralised by positive background charges.) The $2 k_{F}$ oscillatory part of the density comes from the interference between the left- and right-movers. For a homogeneous wire, the interaction 
between the 'slow $\partial_{x} \theta$ mode' and the 'fast $2 k_{F}$ mode' averages out and we only need to take the slow modes into account. However, the oscillatory part can play a role in charging impurities.

To incorporate voltage biases at the ends of the wires, we need to put appropriate boundary conditions on the fermion densities [9]. The left reservoirs at $x=-L / 2$, at voltages $U_{1}^{A}$ inject right-moving charges

$$
\rho_{R}^{A 0}(-L / 2)=e U_{1}^{A} / 2 \pi \hbar v_{F}
$$

where $1 / 2 \pi \hbar v_{F}$ is the density of states for right-moving particles, and similarly the right reservoirs introduce left-moving charges,

$$
\rho_{L}^{A 0}(L / 2)=e U_{2}^{A} / 2 \pi \hbar v_{F} .
$$

(There is some subtlety in bosonisation for finite lengths, but we ignore it under the assumption that $L$ is much larger than any other length scale in the problem and can be effectively taken to be infinite.) We assume that the outgoing particles enter the reservoirs with no reflection. In the presence of intra-wire interactions and inter-wire couplings, the densities of the right and left-moving particles in the wire are not fixed by the external biases, but rather have to be dynamically and self-consistently determined.

In the absence of any impurities and when there is no inter-wire coupling, the problem reduces to that of a single quantum wire for each of the wires. Hence, let us briefly review the properties of a single wire to set the notation. For a single wire with external potentials $U_{1}$ and $U_{2}$, we can solve for the densities as follows 9]. (All densities are measured with respect to the zero external voltage, non-interacting equilibrium density $\rho=k_{F} / \pi$.) Since the electrons are charged, and have Coulomb repulsions, to increase the density of the electrons in the wire through external potentials costs (Coulomb) energy. This potential energy is neutralised beyond the screening length by a screening backgate. (This can be a metal cylinder surrounding the wire at a distance $R_{s}$.) But between the wire and the gate, a capacitor is formed, whose charging energy is given by

$$
\frac{Q^{2}}{2 C}=\frac{e^{2}}{2 C} \int d x \rho^{2}(x)
$$

This energy is provided by the interaction energy within the wire given by

$$
H_{I}=\frac{1}{2} \int d x d y \rho(x) U(x-y) \rho(y)
$$

where $U(x-y)$ is the Coulomb interaction, which in turn is related to the Luttinger liquid parameter $g$. As a concrete example, let us take the screened $U(x-y)$ to be of the form 14

$$
\begin{aligned}
U(x-y) & =\frac{U_{0}}{2 R_{s}} \exp \left(-|x-y| / R_{s}\right) \\
\Longrightarrow U_{k} & =\frac{U_{0}}{1+R_{s} k^{2}} .
\end{aligned}
$$


The Luttinger liquid parameter $g$ is related to $U_{0}$ as

$$
g=\left(1+U_{0} / \pi \hbar v_{F}\right)^{-1 / 2}
$$

For length scales larger than the screening length $R_{s}$, the Coulomb interaction is just a $\delta$-function, so that the interaction Hamiltonian becomes

$$
H_{I}=\frac{U_{0}}{2} \int d x \rho^{2}(x)
$$

Comparing Eqs.(6) and (10), we see that the capacitance per unit length is given by $C=$ $e^{2} / U_{0}$. The electrostatic potential drop $\phi(x)$ between the wire and the screening gate is given by rewriting the interaction energy as

$$
H_{I}=\frac{e}{2} \int d x \rho(x) \phi(x)
$$

which gives $e \phi(x)=U_{0} \rho(x)$. For free fermions $U_{0}=0$ and there is no potential drop and no need of screening.

When we introduce a single impurity into the system, several effects have to be taken into account. The impurity can act as a resistance impeding the flow of current. However, for an interacting system in one dimension, the voltage drop across the impurity need not be the same as the voltage drop across the two ends of the wire. The external voltage drop ( or the two-terminal voltage) is always given by $U_{1}-U_{2}$. But the voltage drop across the impurity can range from 0 , for impurity strength $\rightarrow 0$, to $\left(1-g^{2}\right)\left(U_{1}-U_{2}\right)$ for impurity strength $\rightarrow \infty$. Note that only for $g \rightarrow 0$, the voltage drop across the impurity can be the full two-terminal voltage. This is because for $g \rightarrow 0$, the Coulomb interaction is so strong that there can be no excess charge on the wire.

For our problem, let us first start with uncoupled wires. If $\rho_{L}^{A 0}$ and $\rho_{R}^{A 0}$ are introduced into the wire by the reservoirs, inclusion of the charging energy reduces the density of the particles in the quantum wire by $e \phi \times$ density of states $1 / \pi \hbar v_{F}$. (Remember that the electrons are free to flow out through the outgoing reservoirs.) So the actual densities of the left and right movers in the quantum wires satisfy the relation

$$
\begin{aligned}
\rho_{R}^{A}+\rho_{L}^{A} & =\rho_{R}^{A 0}+\rho_{L}^{A 0}-e \phi / \pi \hbar v_{F} \\
& =g^{2}\left(\rho_{R}^{A 0}+\rho_{L}^{A 0}\right)
\end{aligned}
$$

using Eq.(9). Since the difference of the densities of the right and left movers stays constant, $\left(\rho_{R}^{A}-\rho_{L}^{A}=\rho_{R}^{A 0}-\rho_{L}^{A 0}\right)$, we can solve for $\rho_{R}^{A 0}$ and $\rho_{L}^{A 0}$ in terms of $\rho_{R}^{A}$ and $\rho_{L}^{A}$ as

$$
\begin{aligned}
& \rho_{R}^{A 0}=\frac{\left(1+g^{2}\right)}{2 g^{2}} \rho_{R}^{A}+\frac{\left(1-g^{2}\right)}{2 g^{2}} \rho_{L}^{A} \\
& \rho_{L}^{A 0}=\frac{\left(1-g^{2}\right)}{2 g^{2}} \rho_{R}^{A}+\frac{\left(1+g^{2}\right)}{2 g^{2}} \rho_{L}^{A} .
\end{aligned}
$$


Hence, we now see that the external voltages can be written as boundary conditions for the $\theta^{A}$ fields 9 ,

$$
\begin{aligned}
\left(\frac{1}{g^{2}} \partial_{x}+\frac{1}{v_{F}} \partial_{t}\right) \theta^{A}(x=-L / 2) & =\frac{e U_{1}^{A}}{\sqrt{\pi} \hbar v_{F}} \\
\left(\frac{1}{g^{2}} \partial_{x}-\frac{1}{v_{F}} \partial_{t}\right) \theta^{A}(x=L / 2) & =\frac{e U_{2}^{A}}{\sqrt{\pi} \hbar v_{F}}
\end{aligned}
$$

Let us now consider couplings between the two wires. Provided $d$ is large enough, the couplings at $-d$ and $d$ do not interfere. We can have density- density couplings and single particle tunnelings at the two contacts. For the case of a single contact, the density-density and single-particle tunnelings have already been studied for a single crossing in Ref. [12]. They explicitly showed that for $g<1$, the effects of single-particle tunneling can be captured by renormalisation of the electro-static density-density couplings. Hence, it is only necessary to keep the density-density couplings. For the two crossing case, we have density-density electrostatic interactions at two points given by

$$
V_{\mathrm{den}}=\lambda_{1} \rho^{1}(-d) \rho^{2}(-d)+\lambda_{2} \rho^{1}(d) \rho^{2}(d)
$$

Rewriting this interaction in terms of the phase fields using Eq. (3), (only the 'fast modes') and keeping only terms with scaling dimension $\leq 1$, we obtain

$$
V_{\mathrm{den}}=\lambda_{1} \cos \left(\sqrt{4 \pi} \theta_{1}(-d)\right) \cos \left(\sqrt{4 \pi} \theta_{2}(-d)\right)+\lambda_{2} \cos \left(\sqrt{4 \pi} \theta_{1}(d)\right) \cos \left(\sqrt{4 \pi} \theta_{2}(d)\right)
$$

with scaling dimension $\eta=2 g$. ( $\eta=2 g$ because rewriting the Hamiltonian in the standard form with no interactions will require a rescaling of the $\theta$ field by $\sqrt{g}$. ) All other terms are irrelevant and will renormalise to zero in the low energy limit. Obviously, for $g<1 / 2$, this coupling is relevant and will grow at low energies. But unlike for scatterers in a single wire, for $1 / 2<g<1$, this coupling is irrelevant for coupled wires. Thus, for $g=1$, which is the appropriate limit for uncorrelated electrons, we obtain the results from the usual Landauer-Buttiker formalism in the geometry of Fig.(1).

For $1 / 2<g<1$ or weak repulsive interactions in the two wires, $\lambda_{i} \rightarrow 0$ explicitly, and there is perfect transmission independently in each wire at the fixed point. At zero temperature, the I-V characteristics are governed by the exact results given by

$$
I^{A}=\frac{e^{2}}{h} U^{A} .
$$

What happens in the regime where the Luttinger interactions are strong? Surprisingly, for $0<g<1 / 2$ (for strong repulsive interactions), the Hamiltonian $H=H_{0}+V_{\text {den }}$ decouples in terms of the linear combinations of the phase fields given by

$$
\begin{aligned}
\theta^{ \pm} & =\left(\theta^{1} \pm \theta^{2}\right) / \sqrt{2} \\
\text { and } \quad \phi^{ \pm} & =\left(\phi^{1} \pm \phi^{2}\right) / \sqrt{2}
\end{aligned}
$$


into a sum $H_{+}+H_{-}$with

$$
H_{ \pm}=\frac{\hbar v_{F}}{2 g} \int d x\left\{g\left(\partial_{x} \phi_{ \pm}\right)^{2}+g^{-1}\left(\partial_{x} \theta_{ \pm}\right)^{2}\right\} \pm \frac{\lambda_{1}}{2} \cos \left[\sqrt{8 \pi} \theta_{ \pm}(-d)\right] \pm \frac{\lambda_{2}}{2} \cos \left[\sqrt{8 \pi} \theta_{ \pm}(+d)\right]
$$

and the boundary conditions on these fields are given by

$$
\begin{aligned}
\left(\frac{1}{g^{2}} \partial_{x}+\frac{1}{v_{F}} \partial_{t}\right) \theta^{ \pm}(x=-L / 2) & =\frac{\left(e U_{1}^{1} \pm e U_{1}^{2}\right)}{\sqrt{\pi} \hbar v_{F}} \\
\left(\frac{1}{g^{2}} \partial_{x}-\frac{1}{v_{F}} \partial_{t}\right) \theta^{ \pm}(x=L / 2) & =\frac{\left(e U_{2}^{1} \pm e U_{2}^{2}\right)}{\sqrt{\pi} \hbar v_{F}}
\end{aligned}
$$

Hence, we have two decoupled Hamiltonions, each with Luttinger interactions $\tilde{g}=2 g$ (because rescaling the kinetic term gives the dimension of the barrier terms as $\eta=2 g=\tilde{g}$ and we do not rescale the sound velocity $v=v_{F} / g$ ) and each with two scatterers at the positions $\pm d$. So just like the single crossing case considered by Komnik and Egger [12], the doubly crossed Luttinger liquid also gets mapped into two single wire problems (albeit with twice the interaction strength) and with two potential scatterers.

The case for $g=1 / 2,(\tilde{g}=1)$ can now be trivially addressed. It corresponds to a system of totally uncorrelated fermions with two barriers at $\pm d$. For a single barrier, it is easy to see by refermionising that the transmission coefficient is given by

$$
T=\frac{1}{1+(\lambda / 2)^{2}}
$$

This agrees with the result in Ref. 12 that for a single barrier, the $I-V$ characteristic for the two wires is given by

$$
I^{A}=\frac{e^{2}}{h} T U^{A}=\frac{e^{2}}{h\left(1+(\lambda / 2)^{2}\right)} U^{A}
$$

For two barriers, the transmission coefficient depends on whether the scatterers are combined coherently or incoherently [15]. For incoherent scattering,

$$
T(E)=\frac{T_{1} T_{2}}{1-2 \sqrt{R_{1} R_{2}} \cos \theta(E)+R_{1} R_{2}}
$$

where $E$ is the incident energy, $\theta(E)$ is the phase shift acquired in a round trip between the barriers,$T_{i}=\frac{1}{\left(1+\left(\lambda_{i} / 2\right)^{2}\right)}$ are the two transmission coefficients through the two barriers and $R_{i}=1-T_{i}$ are the reflection coefficients. Clearly, depending on the incident energies, the transmission varies and resonant transmission is possible, whenever the denominator becomes small. For incoherent scattering,

$$
T(E)=\frac{T_{1} T_{2}}{1-R_{1} R_{2}}
$$


which is independent of the phase $\theta$. Here, there exists no possibility of resonances.

An interesting point to note in the above analysis is that resonant transmission is possible, both for symmetric barriers ( in which case $T_{1}=T_{2}$ and the transmission is perfect, $\mathrm{T}\left(E_{\mathrm{res}}\right)$ $=1$ ), and for asymmetric barriers, where the transmission is less than perfect.

For the correlated system, $(g \neq 1 / 2)$, this problem (without the external potentials) was originally addressed in the pioneering Kane-Fisher[0] paper, where it was shown that although in the strong interaction regime, the scattering potentials grow at low energies, and prevent transport, for two scatterers, it was possible to have resonant scattering by tuning a single parameter, either the incident energy or a backgate voltage. In the large barrier limit, the charge on the island between the two barriers is fixed and there will be an energy barrier to add another electron. However, by tuning a gate voltage, we can make the energy cost to add another electron vanish. Hence, as a function of the gate voltage, one can get resonances. The resonance condition is fixed by having $E(n)=E(n+1)$, where $E(n)$ is the energy of the system with charge ne on the island and is given by

$$
4 e g^{2} \Delta \phi_{G}^{ \pm}=e \tilde{g}^{2} \Delta \phi_{g}^{ \pm}=\pi \hbar v_{F} / 2 d
$$

where $\Delta \phi_{G}^{ \pm}$is the change in the gate voltage required to move from one resonance to the other for the \pm wires. However, in this picture, the charging of the impurity was ignored. Later work 14 included the effects of charging the impurity. For a single impurity, this effect was not observable, because of the large capacitance between the wire and the screening backgate. However, for two impurities, inclusion of these effects changes the resonance condition, which becomes [14]

$$
4 e g^{2} \Delta \phi_{G}^{ \pm}=\left(2 d / \pi \hbar v_{F}+2 C^{ \pm}(2 g) / e^{2}\right)^{-1}
$$

where $C^{ \pm}(2 g)=C_{f r}^{ \pm}(2 g)+C_{2 k_{F}}^{ \pm}(2 g) . \quad C_{f r}^{ \pm}=Q / U$ is the finite range capacitance due to the total antisymmetric charge density on the wire in the presence of the impurity. (The symmetric part of the charge density exists even in the absence of the impurity and as explained earlier is due to imperfect screening.) $C_{2 k_{F}}^{ \pm}$, on the other hand, comes from the $2 k_{F}$ component of $\rho$ in Eq.(3) and can be finite even for a zero range $\left(R_{s}=0\right)$ Luttinger liquid. The total capacitance is the sum of the two contributions. We see that inclusion of the contribution of the capacitance decreases the spacing of the gate voltage at which one gets resonances. Note that the capacitances depend on $2 g$ now instead of $g$ for a single wire. Hence, the resonance spacing changes from that of the single wire. However, since there is no dependence on the external potentials, $C^{+}=C^{-}$. Hence, $\Delta \phi_{G}^{+}=\Delta \phi_{G}^{-}=\Delta \phi_{G}$, which leads to the following very interesting consequence, for coupled wires with symmetric inter-wire couplings and zero external bias, -

$$
\begin{aligned}
\Delta \phi_{G}^{1} & =\Delta \phi_{G}^{+}+\Delta \phi_{G}^{-}=2 \Delta \phi_{G} \\
\Delta \phi_{G}^{2} & =\Delta \phi_{G}^{+}-\Delta \phi_{G}^{-}=0
\end{aligned}
$$

-, i.e., a single gate voltage is sufficient to have resonant transmission in both wires.

What happens when we include finite external potential differences between the two ends of the quantum wire? The correct way 16 to address the problem is to solve the 
Hamiltonian with two scatterers given in Eq.(19) with the boundary conditions in Eq.(20). However, we may use the argument that the effect of the external bias serves to tilt the potential between the barriers. Hence, even if we originally start with symmetric barriers, the external potential bias makes them asymmetric. Thus, the problem of a quantum wire with external potential bias and two scatterers, is analagous to the problem without external bias, but with asymmetric scatterers. In this case, it is not possible to get a zero-energy bound state, which was what was needed to tune the resonance for symmetric barriers. Thus, using the argument in Ref. [7] we see that, at least for zero temperature, for coupled wires with an external bias, there is no resonance, and the system is insulating.

For weak barriers, even the capacitances do have dependences on the external potentials. Moreover, the strong barrier assumption that the potential drop across each barrier is equal to the two-terminal voltage across the barrier, that was used to derive the formulae for $C_{f r}$ and $C_{2 k_{F}}$ in Refs. [14] and [8] is no longer valid. The explicit four terminal voltage drops across the barriers $V_{1}$ and $V_{2}$ have to be computed $[9]$ and used in calculating the capacitances, but that is beyond the scope of this paper 16].

We can generalise this result to the case of $N$ crossings of two wires. It is easy to see that the same definition of the + and - channels as the sum and difference of the fields in the two wires, maps the model to two decoupled wires with coupling strength $2 g$ and $N$ potential scatterers. Naively, one may expect that it would be hard to get resonances, since the scattering at each potential would be incoherent. Hence, the system should be opaque to transmission. However, if the crossings are perfectly symmetric and periodic, we expect the equivalent of Bloch states and complete conductivity for specific incident energies or equivalently, specific tuned values of a single gate voltage[16].

The other generalisation that one can consider is what happens if three or more wires meet at a point. Here, again, if we assume density-density couplings at a point, a naive generalisation of the interaction term shows that the interaction potential becomes irrelevant except when $3 g<1$, or more generally when $m$ wires meet a point, except when $m g<1$. For this very strong interaction region, a similar analysis can be attempted. However, the simplification of mapping the model to two decoupled wires with interactions does not go through. Moreover, this geometry is less physically relevant, unless it is possible to fabricate several wires to make contact at a point.

To conclude, we emphasize the new results in this paper. We have shown that transport in two wires crossing at two points remains unaffected by the crossings for $g>1 / 2$. This is an extension of the earlier result for single crossing in Ref. 12]. For $g<1 / 2$, we map the problem to two decoupled wires with two impurities, again following Ref.[12], where it was done for a single crossing. For the decoupled wires, we show that the resonance condition changes, since the coupling strength is now $2 g$ instead of $g$. The $g=1 / 2$ case has uncorrelated fermions in the two wires and the standard analysis for resonance transmission through a double barrier applies [15]. For symmetrically coupled unbiased wires with correlated electrons, $(g<1 / 2)$, we show that a single gate voltage suffices to tune the resonance condition in both the wires and have maximal transmission. However, for asymmetric couplings between the wires or for wires with external voltages, there is no transmission, except when $g=1 / 2$, in which 
case, there does exist resonant transmission, although not perfect. Finally, we argue that for symmetric crossings at periodic lengths, it is still possible to have resonant tunneling tuned by a single gate voltage.

\section{Acknowledgments}

PD thanks C.S.I.R. (India) for financial support. Both of us thank the Abdus Salam ICTP for hospitality during the course of this work. We would also like to thank the workshop held at Trieste on 'Strongly Correlated Electron Systems' for providing the inspiration.

\section{References}

[1] J. M. Luttinger, J. Math. Phys. 15, 609 (1963).

[2] P. W. Anderson, Science 235, 1196 (1987).

[3] S. Tarucha, T. Honda and T. Saku, Solid State Comm. 94, 413 (1995); A. Yacoby et al, Phys. Rev. Lett.77, 4612 (1996).

[4] A. M. Chang et al, Phys. Rev. Lett.77, 2538 (1996).

[5] S. J. Tan et al, Nature 386, 474 (1997); R. Egger and A. O. Gogolin, Phys. Rev. Lett.79, 5082 (1997); C. L. Kane, L. Balents and M. P. A. Fisher, ibid 79, 5086 (1997).

[6] J. Voit, Rep. Prog. Phys. 57, 977 (1995).

[7] C. L. Kane and M. P. A. Fisher, Phys. Rev. B46, 15,233 (1992).

[8] D. L. Maslov and M. Stone, Phys. Rev. B52, R5539 (1995); R. Egger and H. Grabert, Phys. Rev. Lett.77, 538 (1996); ibid 80, 2255 (1998) [Erratum]; A. Furusaki and N. Nagoasa, Phys. Rev. B54, R5239 (1996).

[9] R. Egger and H. Grabert, cond-mat/9805268.

[10] F. Guinea, Phys. Rev. B32, 7518 (1985); C. de C. Chamon, D. E. Freed and X. G. Wen, Phys. Rev. B53, 4033 (1996).

[11] P. Fendley, A. W. W. Ludwig and H. Saleur, Phys. Rev. B52, 8934 (1995).

[12] A. Komnik and R. Egger, Phys. Rev. Lett.80, 2881 (1998).

[13] For a detailed comparison of the various bosonisation schemes, see J. von Delft and H. Schoeller, cond-mat/9805275. 
[14] R. Egger and H. Grabert, cond-mat/9701057.

[15] S. Datta, Electronic Transport in Mesoscopic Systems, Chapter 6, Cambridge University Press, 1995.

[16] P. Durganandini and S. Rao, work in progress. 